\title{
Depletion of circ-BIRC6, a circular RNA, suppresses non-small cell lung cancer progression by targeting miR-4491
}

\author{
Zhu Jin, Baoan Gao*, Yuan Gong, Li Guan \\ Institute of Respiratory Disease, China Three Gorges University, Yichang Central People's Hospital, Yichang, Hubei, China.
}

SUMMARY Circular RNAs (circRNAs) are non-coding RNAs molecules consisting of a covalently closed continuous loop which have no 5'-3' polarity and contain no polyA tail. Accumulating evidence demonstrates that circRNAs are involved in the initiation and progression of human malignancies. In this study, we explored the expression profile and regulatory role of circ-baculoviral IAP repeatcontaining 6 (circ-BIRC6), a circular RNA, in malignant behaviors in non-small cell lung cancer (NSCLC). Expression levels of circ-BIRC6 and miR-4491 were examined in NSCLC patient samples and cell lines using quantitative real time PCR (qRT-PCR). In vitro roles of circ-BIRC6 knockdown on cell viability, colony formation, and apoptosis were assessed using the CCK-8, colony formation assay, and flow cytometry, respectively. The interactions between circ-BIRC6 and miR-449 were assessed using luciferase reporter and qRT-PCR assays. The in vivo role of circ-BIRC6 knockdown on tumor growth and apoptosis was evaluated in a xenograft mouse model of NSCLC. We found that expression levels of circ-BIRC6 in NSCLC patient samples and cell lines were elevated. Small interfering RNA (siRNA)-mediated circ-BIRC6 knockdown suppressed cell proliferation, colony formation, migration and invasion, and promoted apoptosis in NCI-H460 and A549 cells. In addition, miR-4491 was identified as a tumor-suppressor miRNA in NSCLC and circ-BIRC6 functions as a molecular sponge for miR-4491. Furthermore, circ-BIRC6 knockdown suppressed Wnt2B/ $\beta$-catenin pathway. In vivo assay showed that depletion of circ-BIRC6 suppressed tumor growth, enhanced apoptosis, and decreased miR-4491 levels in a mouse xenograft model. These findings demonstrate that circ-BIRC6 functions as a critical regulator of proliferation and apoptosis via binding to and negatively regulating miR-4491, suggesting that circ-BIRC6 might be a potential target for treatment of NSCLC.

Keywords non-small cell lung cancer, Circular RNA, Circ-BIRC6, MiR-4491

\section{Introduction}

Non-small cell lung cancer (NSCLC) is the leading cause of cancer-related deaths. Although great improvement has been achieved in cancer therapy, approximately $40 \%$ of NSCLC cases have advanced-stage disease with a five-year survival rate of approximately $2 \%$ $(1,2)$. Therefore, further understanding of the molecular mechanisms and development of novel therapies with enhanced specificity are immediately needed for the management of NSCLC.

Circular RNAs (circRNAs), a novel type of RNA, are a special group of non-coding endogenous RNAs molecules, consisting of at least a few hundred nucleotides (3). According to previous studies, most circRNAs are noncoding RNAs while only a few proteincoding circRNAs have been reported (4). Accumulating evidence supports the diverse roles of circRNAs in cellular behavior, including growth, apoptosis, migration, and differentiation. In addition, circRNAs play critical roles in the initiation and progression of human malignancies (5-7). Particularly, circ-BIRC6 (ID hsa circ_0003288) is located on chromosome 2 (3270370232718734 ) and is generated by back-splicing of the BIRC6 transcript (NM_016252). It has been reported that levels of circ-BIRC6 are upregulated in hepatocellular carcinoma (HCC), and depletion of circ-BIRC6 could suppress cell growth, migration, and invasion, representing a potential prognostic factor and therapeutic target for HCC (8). As is well acknowledged, circRNAs regulate gene expression at the transcriptional or posttranscriptional level by binding to microRNAs (miRNAs) or other molecules $(9,10)$. Mechanically, circ-BIRC6 is reported to function as an oncogenic gene via sponging miR-3918 in HCC (8). Although a close relationship between circRNAs and NSCLC has been reported, the 
specific mechanisms by which circRNAs may regulate carcinogenesis and progression of NSCLC remains largely unknown (11). Herein, our study explored the expression pattern of circ-BIRC6 and its effects on the malignant behaviors in NSCLC.

\section{Materials and Methods}

\subsection{Patient samples}

NSCLC tissues and paired adjacent noncancerous lung tissues $(n=30)$ were obtained in the Yichang Central People's Hospital. NSCLC patients were pathologically diagnosed and received no chemotherapy nor radiotherapy before tissue sampling. The samples were immediately frozen and stored in liquid nitrogen. This study was approved by the Ethics Committee of Yichang Central People's Hospital and written informed consent was obtained from all participants.

\subsection{Cell culture}

Human lung cancer cell lines, including NCI-H460 and A549, were obtained from the Shanghai Institute of Biological Sciences, Chinese Academy of Sciences (CAS, Shanghai, China). Cells were maintained in Dulbecco's modified Eagle's medium (DMEM) supplemented with 10\% fetal bovine serum (FBS) (Gibco), $100 \mu \mathrm{g} /$ $\mathrm{mL}$ streptomycin, and $100 \mu \mathrm{g} / \mathrm{mL}$ penicillin (Hyclone, Logan, UT, USA). Cells were maintained at $37^{\circ} \mathrm{C}$ in a humidified atmosphere containing $5 \% \mathrm{CO}_{2}$.

\subsection{Cell viability assay}

CCK-8 assay was performed for determining cell viability, using the CCK-8 assay kit according to the manufacturer's protocol. In brief, cells were seeded into 96-well plates and transfected with negative control construct or target gene-manipulated construct. The cell viability in each well was determined by adding 10 $\mu$ Lof CCK- 8 solution. After further incubation at $37^{\circ} \mathrm{C}$ for $2 \mathrm{~h}$, absorbance was measured using an ELISA reader at a wavelength of $450 \mathrm{~nm}$.

\subsection{Cell transfection}

Cells were plated in 6-well plates and transfected with siRNA for circ-BIRC6 and negative control (si$\mathrm{NC}$ ), according to the manufacturer's instructions of Lipofectamine 2000 (Invitrogen, Carlsbad, CA, USA). Culture medium was replaced six hours after transfection. The interference sequences were circ-BIRC6 siRNA, 5'- CUGAAAGGUUCUUGCACGCAU-3'. Scramble siRNA (5'- GACUUUCGUUCUUGCACGCAU-3') was used as a negative control.

\subsection{Quantitative real-time PCR (qRT-PCR)}

Total RNA was extracted from cultured cells using Trizol reagent. The isolated RNA was reverse transcribed into cDNA, which was used as a template to perform qRT-PCR on an ABI 7900 system. The transcript levels of indicated genes were determined using the $2-\Delta \Delta \mathrm{Ct}$. Primers used in the study were as follows: circ-BIRC6, 5'-TGAAAGGTTCTTGCACGCAT-3' and 5'-GCTGGGG T TCGT TCACAATC-3'; $\beta$-actin, 5'-CACAGAGCCTCGCCTTTGCC-3'; 5'-ACCCATGCCCACCATCACG-3'; miR-4491, 5'-GT TCTGCGTCCCAGGCAGT-3'; 5'-GCTAACGGCTGA TGTGTCCA-3'; U6, 5'-CGAGCACAGAATCGCTTCA -3'; 5'-CTCGCTTCGGCAGCACATAT-3'. U6 levels were used to normalize miR-4491 expression. $\beta$-actin was endogenous control for circ-BIRC6. All reactions were performed in triplicates.

\subsection{Colony formation assay}

Tumor cells were plated at $1 \times 10^{6}$ cells/plate in a 100 $\mathrm{mm}$ culture dish one day before transfection. The next day, cells were transfected with negative control construct or target gene-manipulated construct. The surviving colonies were stained with crystal violet and visible colonies were counted using an automated colony-counting system (Media Cybernetics, Inc., Bethesda, MD, USA).

\subsection{Flow cytometry}

Cell apoptosis was analyzed using annexin V/PI apoptosis kit (Invitrogen, USA) according to the manufacturer's instruction. In brief, cells were seeded in a 6-well plate and transfected with the siRNA negative control or siRNA against circ-BIRC6. The cells from each group were washed with ice-cold PBS, resuspended in $100 \mu \mathrm{L}$ binding buffer, and stained with annexin V-FITC and PI. Apoptosis of samples were then analyzed by flow cytometry (FACSCalibur, BD Biosciences, San Jose, CA, USA).

\subsection{Terminal deoxynucleotidyl transferase d-UTP nick} end labelling (TUNEL) staining

Cell apoptosis was analyzed by TUNEL assay using an in-situ cell death detection kit (Roche, Mannheim, Germany) according to the manufacturer's instruction. In brief, cells were fixed in 4\% paraformaldehyde, incubated in $3 \% \mathrm{H}_{2} \mathrm{O}_{2}$, permeabilized with $0.5 \%$ Triton $\mathrm{X}-100$, and then incubated in the TUNEL reaction mixture. Sections were rinsed, stained with DAPI, and observed under a fluorescence microscopy.

\subsection{Transwell assays}

Transwell assay was used to determine the invasion and migration of cells. For tumor invasion assay, Transwell 
chambers (Costar, Cambridge, MA, USA) were coated with Matrigel on the upper surface. Cells were suspended in serum-free medium in the upper chamber, and lower chamber was added with medium containing $10 \%$ FBS. Forty-eight hours later, cells in upper chambers were removed and stained to detect the invaded cells. The migration assay was performed with the same procedure in Transwell chambers without Matrigel. The numbers of migrated and invaded cells were calculated.

\subsection{Luciferase reporter assay}

Cells were co-transfected with luciferase reporter plasmids carrying circ-BIRC6 wt or circ-BIRC6 mut, and miR-4491 mimics or miR-NC plasmids using Lipofectamine 2000. Then, firefly and Renilla luciferase activities were measured by the Dual-Luciferase Reporter Assay System Kit (Promega) to analyze the interaction between circ-BIRC6 and miR-4491.

\subsection{Western blotting}

Cultured cells were lysed in cell lysis buffer and protein samples were separated by SDS-PAGE. After separation, proteins were transferred onto nitrocellulose membranes (Millipore, Billerica, MA, USA) followed by incubation with primary antibodies anti-bax (dilution 1:2,000, Santa Cruz, USA), Wnt2B (dilution 1:2,000, Santa Cruz, USA), $\beta$-catenin (dilution 1:2,000, Santa Cruz, USA) and anti-bcl-2 (dilution 1:2,000, Santa Cruz, USA), overnight at $4^{\circ} \mathrm{C}$. After washing in PBS, the membranes were incubated with horseradish peroxidase (HRP)-labeled secondary antibody $(1: 5,000)$ at room temperature for $1 \mathrm{~h}$. The membranes were then washed in PBS for three times, and protein bands were detected using the enhanced chemiluminescence (ECL) kit (Pierce, Rockford, IL, USA). Protein bands were captured using the Leica Image Processing system and processed using Image $\mathbf{J}$ software (National Institutes of Health, USA), and their intensity was normalized to that of the band for $\beta$-actin $(1: 2,000)$.

\subsection{Xenograft model}

Female BALB/c mice (4-6 weeks old) were purchased from Shanghai Slac Laboratory Animal Co. Ltd. (Shanghai, China). All procedures for animal studies were approved by the ethics committee of Yichang Central People's Hospital. Wild-type or circ-BIRC6knockdown tumor cells were injected subcutaneously into nude mice ( $n=5$ per group). Subsequently, mice in each group were intraperitoneally injected with cisplatin (20 mg/kg body weight) and tumor formation was observed. Tumors were measured once a week for five weeks followed by determination of tumor volume and tumor weight. For tumor volume, the following formula was used: $\mathrm{V}=\pi / 6 \times \mathrm{L} \times \mathrm{W} 2$, where $\mathrm{V}=$ volume $(\mathrm{mm} 3)$, $\mathrm{L}=$ length $(\mathrm{mm})$, and $\mathrm{W}=$ width $(\mathrm{mm})$.

\subsection{Statistical analysis}

Data are expressed as mean \pm standard deviation (SD). Differences between two groups were compared with Student test, while differences between multiple groups were compared using ANOVA followed by Tukey tests. Results with a $P$ value $<0.05$ were considered statistically significant.

\section{Results}

3.1. circ-BIRC6 is upregulated in NSCLC tissues and cell lines

Expression profile of circ-BIRC6 in NSCLC patient samples and cell lines was determined using quantitative real time PCR (qRT-PCR). Data showed that the expression of circ-BIRC6 was significantly upregulated in NSCLC tissues compared to the adjacent normal tissues (Figure 1A). Additionally, levels of circ-BIRC6 in two NSCLC cell lines, including NCI-H460 and A549,
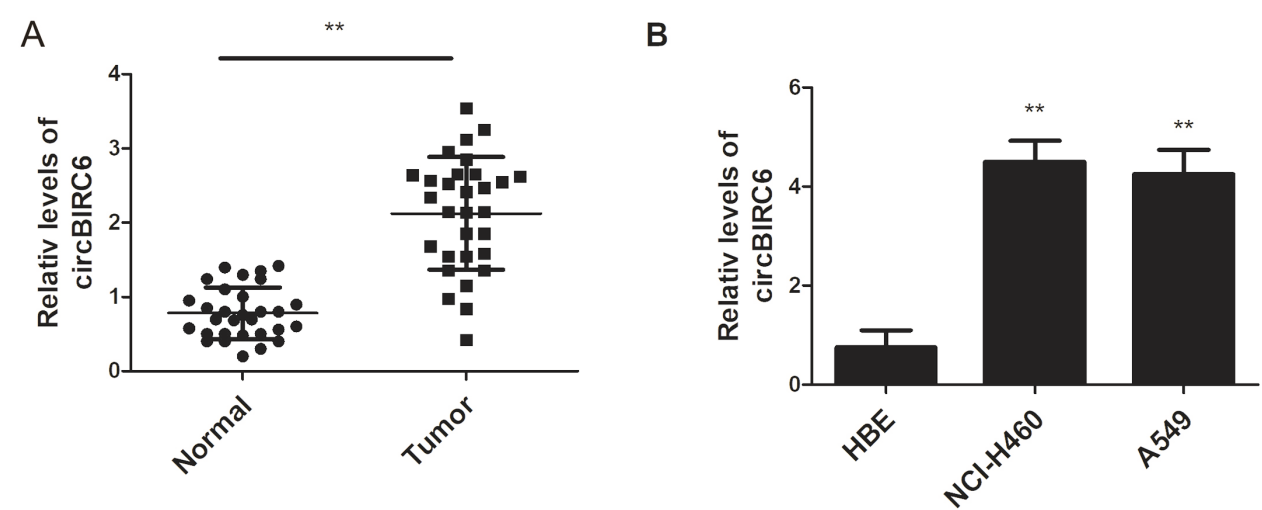

Figure 1. circ-BIRC6 is upregulated in NSCLC tissues and cell lines. Relative expression of circ-BIRC6 in 30 pairs of NSCLC and normal tissue was determined by qRT-PCR (A). In addition, expression of circ-BIRC6 was detected in NCI-H460, A549 and human bronchial epithelial cells (B). Results are expressed as mean $\pm \mathrm{SD},{ }^{* *} P<0.01,{ }^{* * *} P<0.001$. 
and the human bronchial epithelial cell line (HBE), were detected. Consistently, we found that circ-BIRC6 was overexpressed in NCI-H460 and A549 cells compared to HBE cells (Figure 1B). These results imply a possible oncogenic role of circ-BIRC6 in the progression of NSCLC cells.

3.2. circ-BIRC6 depletion suppresses malignant behaviors of NSCLC cells

To explore the potential oncogenic roles of circ-BIRC6 in vitro, NCI-H460 and A549 cells transfected with designed siRNA specifically targeting circ-BIRC6. Transfection with this siRNA effectively silenced circBIRC6 levels in NCI-H460 and A549 cells (Figure 2A) but had no effect on linear BIRC6 mRNA levels (Figure 2B). Consequently, siRNA-mediated knockdown of circ-BIRC6 remarkably inhibited cell proliferation and colony formation (Figure 2C and D). In addition, we found that depletion of circ-BIRC6 significantly induced apoptosis compared to the scramble control in NCI-H460 and A549 cells, as determined by flow cytometry (Figure 3A and B). On the molecular level, western blot revealed that knockdown of circ-BIRC6 dramatically promoted the expression of bax and suppressed bcl-2 in NCI-H460 and A549 cells (Figure 3C). Furthermore, transwell analysis showed that downregulation of circBIRC6 inhibited migration and invasion of NCI-H460 and A549 cells (Figure 3D) Collectively, these findings demonstrated that knockdown of circ-BIRC6 could exert the anti-tumor properties in NSCLC cells.
3.3. circ-BIRC6 binds directly to miR-4491 in NSCLC cells

To elucidate the underlying mechanism by which circBIRC6 manipulates NSCLC progression, we screened potential targets of circ-BIRC6 using online software Circular RNA Interactome (http://regrna2.mbc.nctu. $e d u . t w /)$ and identified that miR-4491 as a candidate target (Figure 4A). Real-time PCR results showed that circ-BIRC6 depletion led to an obvious increase of miR-4491 in NCI-H460 and A549 cells (Figure 4B). To validate the hypothesis that circ-BIRC6 serves as a miRNA sponge, we generated wild type (wt) circBIRC6 luciferase plasmids containing potential miR4491 binding sites, as well as mutant variants of binding site. Following co-transfection, the luciferase activity was lower in the circ-BIRC6 wt+miR-4491 group than in the si-NC group, while was comparable in circ-BIRC6-mut+miR-4491 group and the si-NC group, in both NCI-H460 and A549 cells (Figure 4C). Taken together, these results demonstrated that circ-BIRC6 can act as a molecular sponge for miR-4491 in NSCLC cells.

3.4. miR-4491 overexpression suppresses cell proliferation in NSCLC

In addition, we explored whether cell viability was affected by miR-4491 overexpression. Firstly, we detected levels of miR-4491 in NSCLC samples and cell lines, as determined using qRT-PCR. Data showed
A

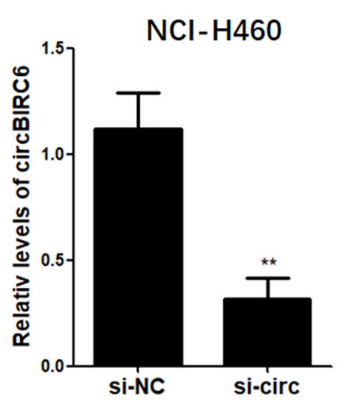

C

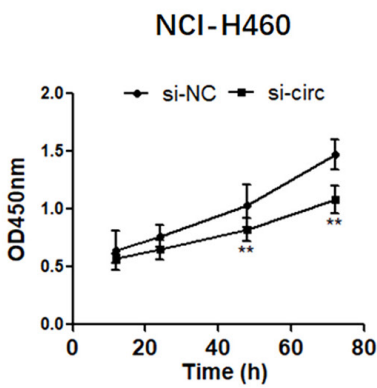

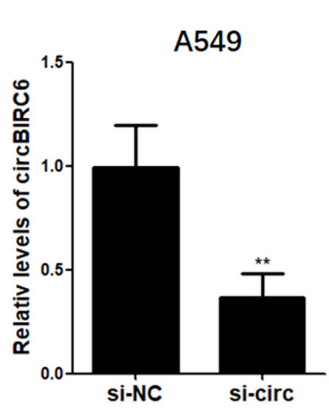

B

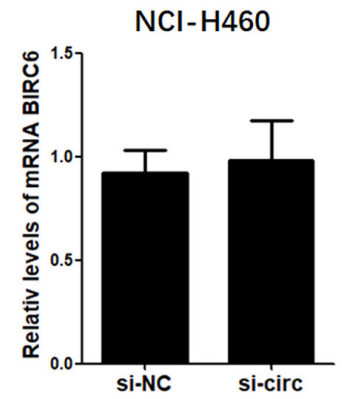

D

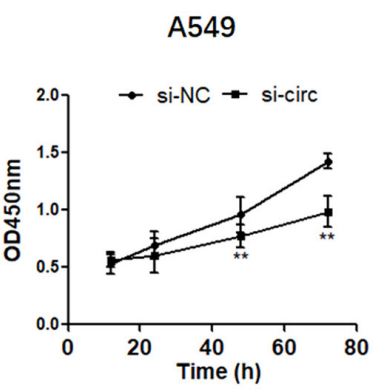

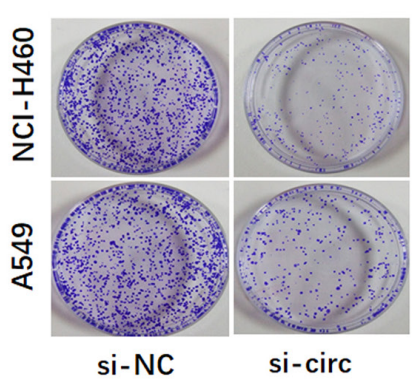

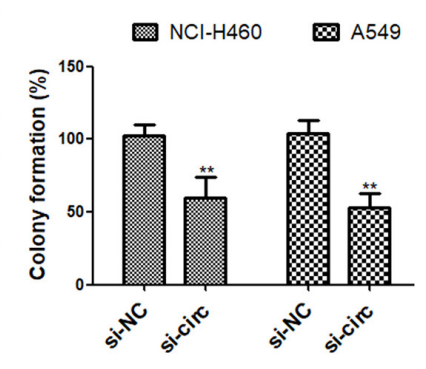

Figure 2. circ-BIRC6 depletion suppresses cell viability and colony formation in NSCLC cells. NCI-H460 and A549 cells were transfected with circ-BIRC6 siRNA or negative control. Levels of circ-BIRC6 and linear BIRC6 mRNA in NCI-H460 and A549 cells were determined using qRT-PCR (A and B). In addition, cell proliferation and colony formation of NCI-H460 and A549 cells transfected with circ-BIRC6 siRNA were detected by CCK-8 assay (C) and colony formation assay (D), respectively. Results are expressed as mean \pm SD, ${ }^{* *} P<0.01$. 


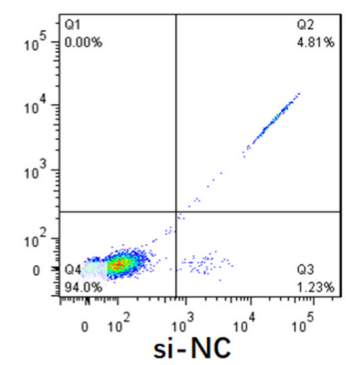

B

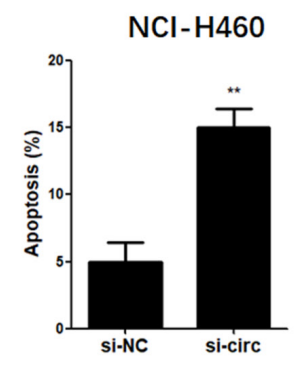

$\mathrm{NCl}-\mathrm{H} 460$

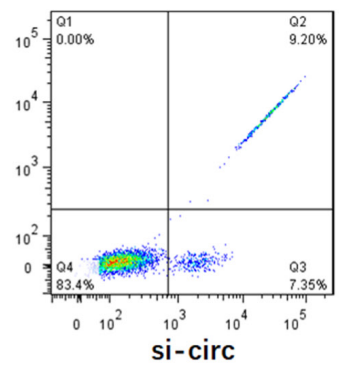

A549

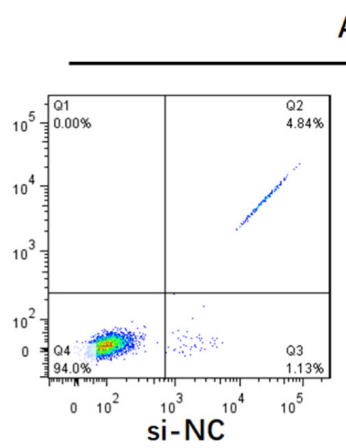

A549

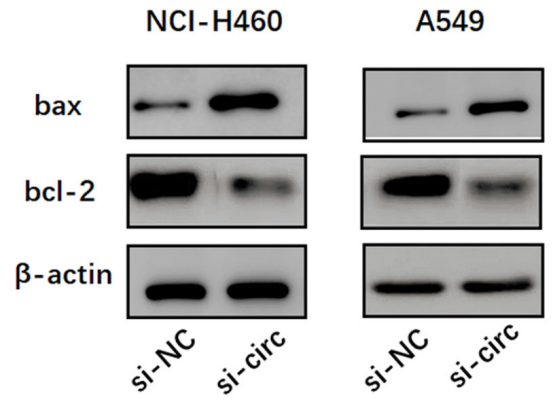

D

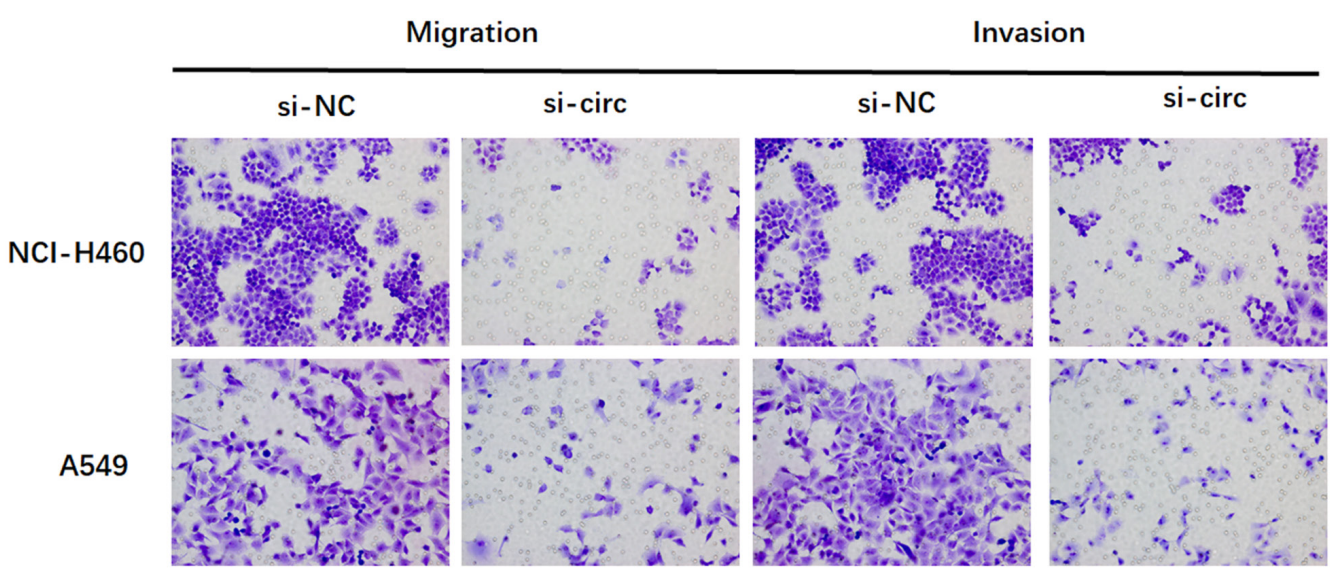

Figure 3. circ-BIRC6 depletion enhances apoptosis in NSCLC cells. NCI-H460 and A549 cells were transfected with circ-BIRC6 siRNA or negative control. Levels of apoptosis rates in NCI-H460 and A549 cells were determined using flow cytometry (A and B). In addition, apoptosisrelated proteins including bax and bcl-2 in NCI-H460 and A549 cells transfected with circ-BIRC6 siRNA were detected by western blot (C). (D) Transwell analysis was conducted to measure the migration and invasion in NCI-H460 and A549 cells transfected with circ-BIRC6 siRNA. Results are expressed as mean $\pm \mathrm{SD},{ }^{* *} P<0.01$.

that miR-4491 was significantly downregulated in NSCLC tissues compared to the adjacent normal tissues (Figure 5A). Consistently, levels of miR-4491 were also decreased in NCI-H460 and A549 cells compared to HBE cells (Figure 5B). Strikingly, CCK-8 assay indicated that NCI-H460 and A549 cells ectopically expressed miR-4491 showed a slower growth rate, which were reversed after co-transfection with circBIRC6 (Figure 5C and D). Furthermore, miR-4491 was predicted to target Wnt2B (Figure 5E). Indeed, downregulation of circ-BIRC6 inhibited the expression of Wnt2B and $\beta$-catenin in NCI-H460 and A549 cells (Figure 5F). Taken together, these data suggested that miR-4491 exerted a tumor suppressor role via regulating $W n t 2 B / \beta$-catenin signaling pathway in NSCLC cells.

\subsection{In vivo effect of circ-BIRC6 knockdown on tumor growth}

To explore the in vivo effect of circ-BIRC6-knockdown on tumor growth, NCI-H460 cells transfected with circBIRC6 siRNA or negative control, were injected into the flank of $\mathrm{BABL} / \mathrm{c}$ nude mice. There was a significant reduction in tumor volume in mice injected with circBIRC6-knockdown cells compared to that in mice 
A circ-BIRC6-wt 5'-GCCGUGACTCCGTCAG-3'
Has-miR-4491 3'---GAUAGUGAGGCACCA-5'
circ-BIRC6-mut 5'-GCCGUGAGCAAGTCAG-3'

C

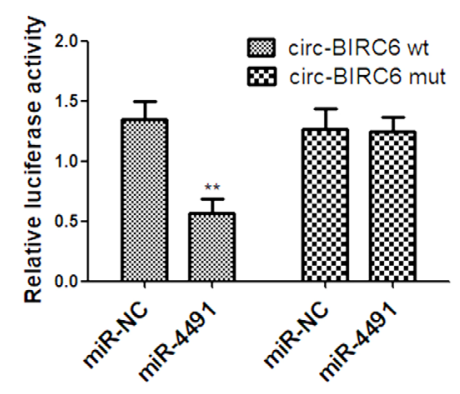

B

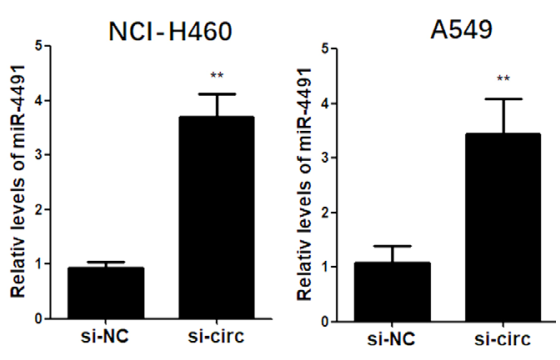

A549

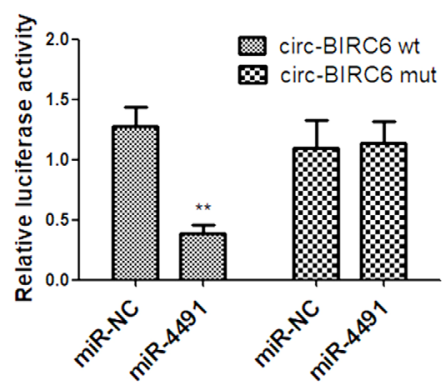

Figure 4. circ-BIRC6 binds directly to miR-4491 in NSCLC cells. NCI-H460 and A549 cells were transfected with circ-BIRC6 siRNA or negative control. Bioinformatics analysis of potential circ-BIRC6/miR-4491 interactions (A). Then, expression of miR-4491 was detected in NCI-H460 and A549 cells transfected with circ-BIRC6 siRNA (B). A dual luciferase reporter plasmid containing circ-BIRC6-wt or circ-BIRC6mut was co-transfected into NCI-H460 and A549 cells along with miR-4491 mimics or miR-NC, and luciferase activities were determined (C). Results are expressed as mean $\pm \mathrm{SD},{ }^{* * *} P<0.01$.

A

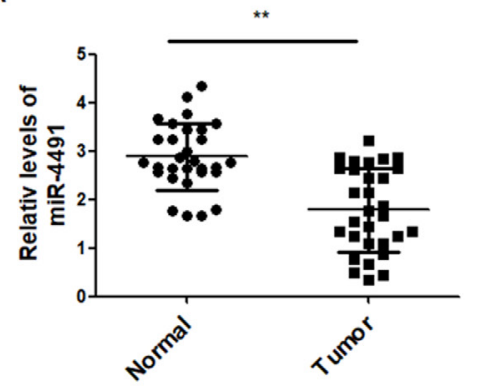

$\mathrm{NCl}-\mathrm{H} 460$

C

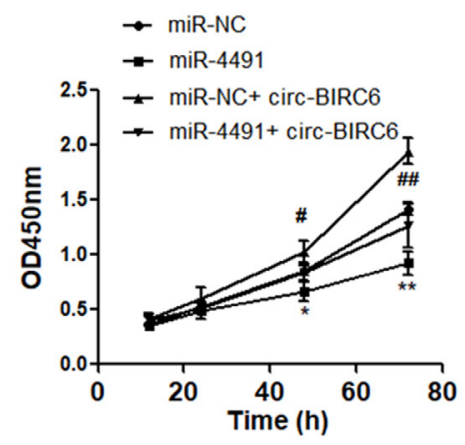

$\mathrm{E}$

'WNT2B 3' UTR 5' ...UUGGCCAGAGGGUCCACAUC... hsa-miR-4491 3' AAAGUGUGGUCAGGUGUAA
B

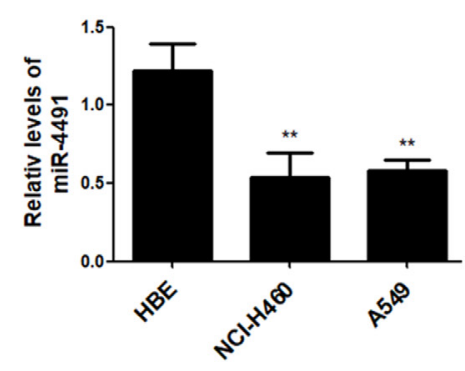

D

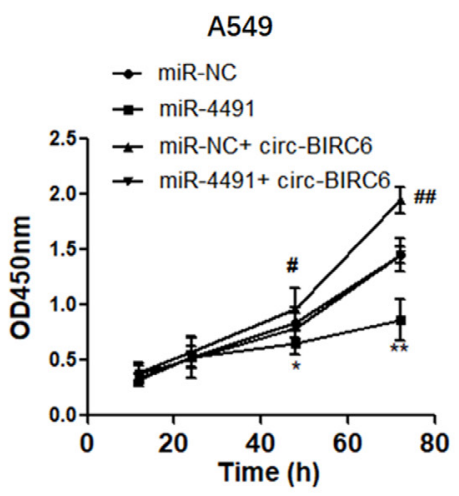

$\mathrm{F}$

$\mathrm{NCl}-\mathrm{H} 460$

A549

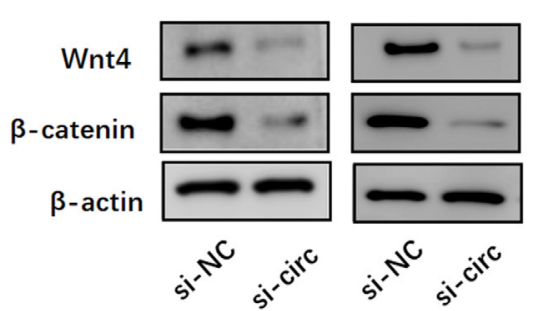

Figure 5. miR-4491 overexpression suppresses cell proliferation in NSCLC. Relative expression of miR-4491 in 30 pairs of NSCLC and normal tissue was determined by qRT-PCR (A). ${ }^{* *} P<0.01$, compared to normal. In addition, expression of miR-4491 was detected in NCI-H460, A549 and human bronchial epithelial cells (B). Results are expressed as mean $\pm \mathrm{SD},{ }^{* *} P<0.01$, compared to HBE cells. In addition, CCK-8 assay was performed to determine the cell proliferation of NCI-H460 (C) and A549 (D) cells transfected with miRNC, miR-4491 mimics, circ-BIRC6 alone miR-4491 mimics combined with circ-BIRC6. (E) Bioinformatics analysis of potential miR-4491/ Wnt2B interactions. (F) Expression of Wnt2B and $\beta$-catenin in NCI-H460 and A549 cells transfected with circBIRC6 siRNA. Results are expressed as mean $\pm \mathrm{SD},{ }^{*} P<0.05,{ }^{* *} P<0.01$ compared to miR-NC. 
A
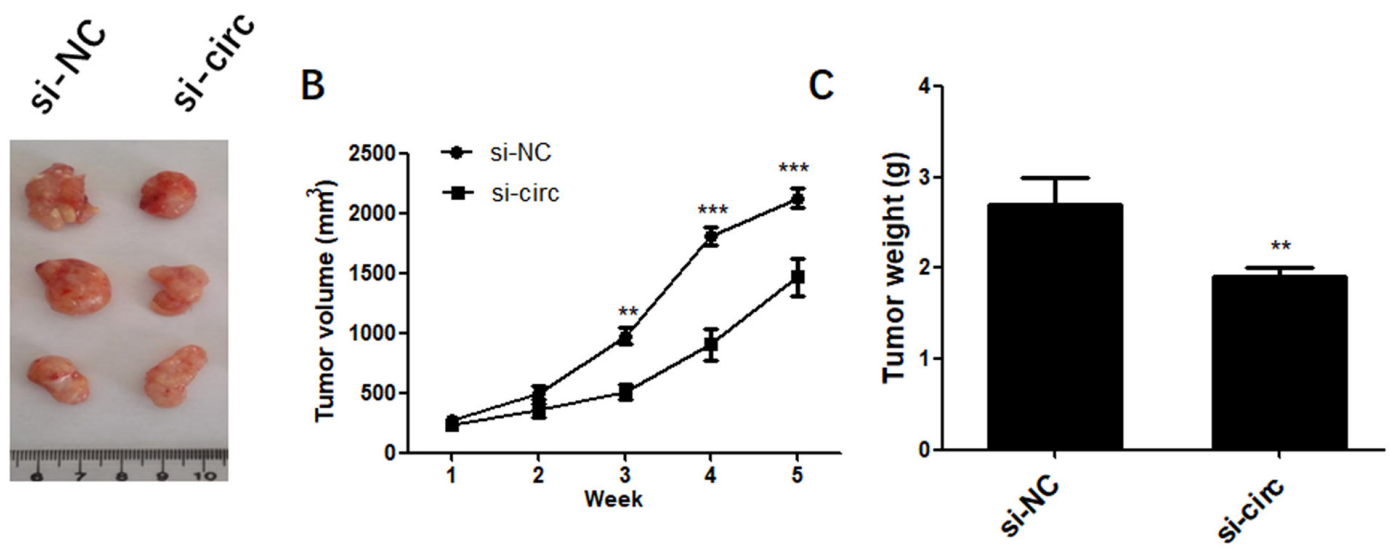

D
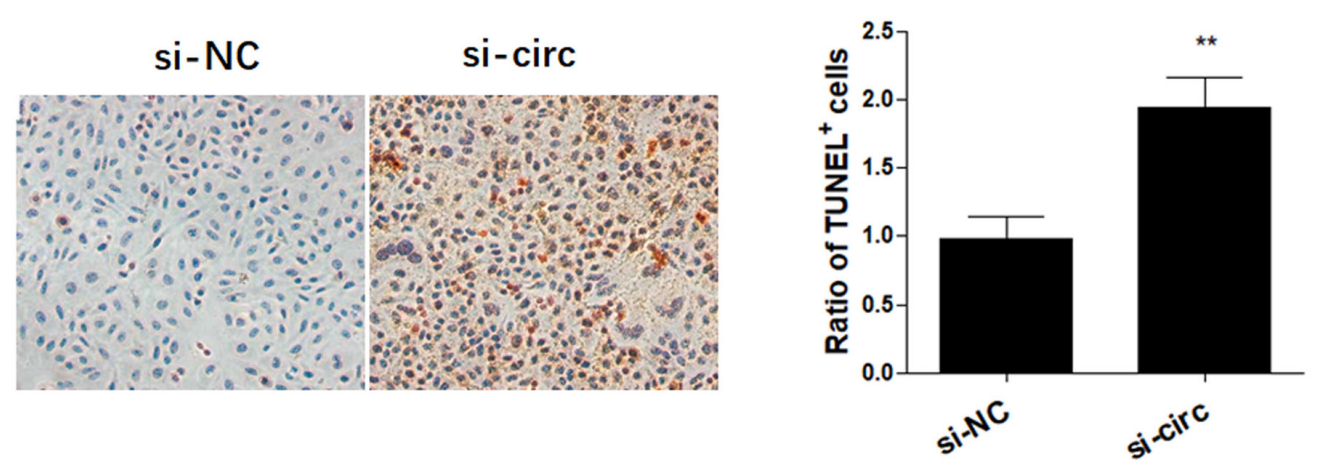

$E$

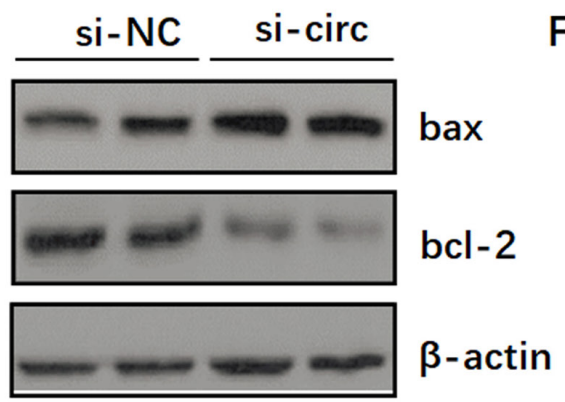

$\mathrm{F}$

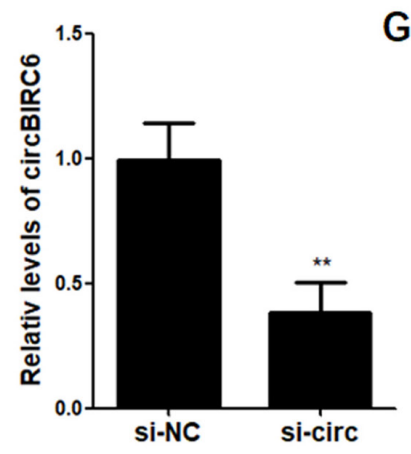

G

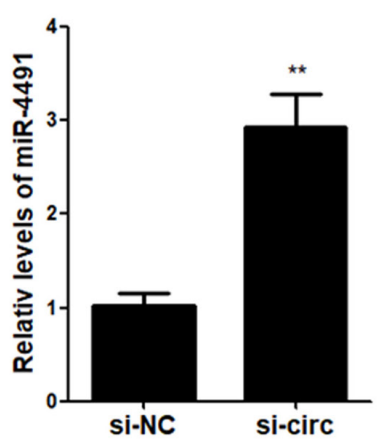

Figure 6. circ-BIRC6 depletion inhibits tumor growth in vivo. NCI-H460 cells were transfected with circ-BIRC6 siRNA or negative control, and injected subcutaneously into the flank of nude mice. Representative images of resected tumors (A). Tumor volume and tumor weight were measured (B and $\mathbf{C}$ ). A TUNEL assay was performed to analyze the induction of apoptosis by circ-BIRC6 knockdown (D). In addition, apoptosisrelated proteins including bax and bcl-2 in resected tumors were detected by western blot (E). Relative expression of circ-BIRC6 (F) and miR4491 (G) in tumors. Results are expressed as mean $\pm \mathrm{SD},{ }^{* *} P<0.01,{ }^{* * * *} P<0.001$.

injected with control cells (Figure 6A-B). In addition, circ-BIRC6 depletion significantly decreased the tumor weight compared to that in control mice (Figure 6C). It was demonstrated by TUNEL assay that apoptosis rate in tumor cells was increased after depletion of circBIRC6 (Figure 6D). Consistently, western blot revealed that knockdown of circ-BIRC6 dramatically promoted the expression of bax and suppressed bcl-2 (Figure $6 \mathrm{E})$. In addition, Moreover, expression of circ-BIRC6 was decreased while miR-4491 was elevated, in circBIRC6 siRNA-derived tumor tissues (Figure 6F and $\mathrm{G}$ ). Collectively, these data indicate that depletion of circBIRC6 could exert a tumor suppressive role in NSCLC in vivo.

\section{Discussion}

NSCLC is the most common malignant tumor, ranking first among the reasons for cancer-related deaths worldwide. Despite therapeutic advances, the prognosis of patients with NSCLC is not satisfactory. Recent studies have demonstrated that circRNAs play critical roles in the pathogenesis and progression of malignancies, representing novel biomarkers and therapeutic targets in the management of NSCLC $(12,13)$.For instance, hsa_circRNA_102958 is significantly in gastric cancer and correlated with TNM stage, representing a novel diagnostic marker for gastric cancer (14). Autophagyassociated circRNA circCDYL promotes breast cancer 
progression via the miR-1275-ATG7/ULK1 axis (15), while circHIPK3 sponges miR-124-3p to promote proliferation and metastasis of lung cancer both in vitro and in vivo (16). Recently, Yang et al. identified high expression of circ-BIRC6 in NSCLC patients and found that it functioned as an oncogenic factor to promote $\mathrm{HCC}$ progression. Mechanistically, circ-BIRC6 promoted HCC cell proliferation, migration, and invasion via sponging miRNA-3918/bcl-2 axis (8). Additionally, RNA-sequencing revealed that 106 differentially expressed circRNAs, including 61 upregulated circRNAs and 45 downregulated circRNAs, in NSCLC cells treated with a small molecule inhibitor XAV939 (11). Herein, we explored the expression pattern of circBIRC6 in lung cancers and the underlying mechanisms in the pathogenesis of NSCLC. Our results detected elevated expression of circ-BIRC6 in patient samples with NSCLC and cell lines, suggesting a possible link between circ-BIRC6 and tumorigenesis in NSCLC. In addition, circ-BIRC6 knockdown decreased cell proliferation, colony formation, and induced apoptosis in NSCLC cells. The in vivo experiments also confirmed the suppressive effects of circ-BIRC6 knockdown on tumor growth.

Amounting evidences have demonstrated that miRNAs play an important role in the initiation and progression of colorectal (17), lung (18), and cervical (19) cancers. circRNAs contribute to initiation and progression of human malignancies by acting as miRNA sponges to regulate gene and protein expression (20). Recently, Chen et al. have shown that circRNA hsa circ_100395 serves as a sponge for miR-1228 to inhibit progression and metastasis of lung cancer (21), while circTP63 functions as a ceRNA to promote lung squamous cell carcinoma progression by sponging miR-873-3p/FOXM1 axis (22). Herein, we identified downregulated expression of miR-4491 in NSCLC samples and cell lines; overexpression of miR-4491 exerted a tumor suppressor role in NSCLC cells. Furthermore, we identified that circ-BIRC6 functions as a molecular sponge for miR-4491. Both in vitro and in vivo assays suggested that circ-BIRC6 negatively regulated the expression of $\mathrm{miR}-4491$, suggesting that circ-BIRC6/miR-4491 interaction mediates the progression of NSCLC. Particularly, we identified that Wnt2B/ $\beta$-catenin signaling pathway was involved in circ-BIRC6-miR-4491-axis. Aberrant activation of the Wnt $2 \mathrm{~B} / \beta$-catenin pathway plays a critical role in tumor initiation, progression, and metastasis of lung cancer (23-25). Our study found that downregulation of circBIRC6 inhibited the expression of Wnt2B and $\beta$-catenin in NCI-H460 and A549 cells.

In conclusion, circ-BIRC6 functions as a critical regulator of growth and apoptosis in NSCLC cells via sponging miR-4491. Our study suggests that circ-BIRC6 might be a novel molecular target for the management of NSCLC.

\section{Funding: None.}

Conflict of Interest: The authors have no conflict of interest to disclose.

\section{References}

1. Rajurkar S, Mambetsariev I, Pharaon R, Leach B, Tan T, Kulkarni P, Salgia R. Non-Small Cell Lung Cancer from Genomics to Therapeutics: A Framework for Community Practice Integration to Arrive at Personalized Therapy Strategies. J Clin Med. 2020; 9:E1870.

2. Torre LA, Siegel RL, Jemal A. Lung cancer statistics. Adv Exp Med Biol. 2016; 893:1-19.

3. Matsui M, Corey DR. Non-coding rnas as drug targets. Nat Rev Drug Discov. 2017; 16:167-179.

4. Patop IL, Wust S, Kadener S. Past, present, and future of circrnas. Embo J. 2019; 38:e100836.

5. Arnaiz E, Sole C, Manterola L, Iparraguirre L, Otaegui D, Lawrie $\mathrm{CH}$. Circrnas and cancer: biomarkers and master regulators. Semin Cancer Biol. 2019; 58:90-99.

6. Kristensen LS, Andersen MS, Stagsted LVW, Ebbesen KK, Hansen TB, Kjems J. The biogenesis, biology and characterization of circular rnas. Nat Rev Genet. 2019; 20:675-691.

7. Qian L, Yu S, Chen Z, Meng Z, Huang S, Wang P. The emerging role of circrnas and their clinical significance in human cancers. Biochim Biophys Acta Rev Cancer. 2018; 1870:247-260.

8. Yang G, Wang X, Liu B, Lu Z, Xu Z, Xiu P, Liu Z, Li J. Circ-birc6, a circular rna, promotes hepatocellular carcinoma progression by targeting the mir-3918/bcl2 axis. Cell Cycle. 2019; 18:976-989.

9. Xiong DD, Dang YW, Lin P, Wen DY, He RQ, Luo DZ, Feng ZB, Chen G. A circrna-mirna-mrna network identification for exploring underlying pathogenesis and therapy strategy of hepatocellular carcinoma. J Transl Med. 2018; 16:220.

10. Di X, Jin X, Li R, Zhao M, Wang K. Circrnas and lung cancer: biomarkers and master regulators. Life Sci. 2019; 220:177-185.

11. Yu H, Xu L, Liu Z, Guo B, Han Z, Xin H. Circ mdm2_000139, circ_atf2_001418, circ_cdc25c_002079, and circ_birc6_001271 are involved in the functions of xav939 in non-small cell lung cancer. Can Respir J. 2019; 2019:9107806.

12. Di X, Jin X, Li R, Zhao M, Wang K. Circrnas and lung cancer: biomarkers and master regulators. Life Sci. 2019; 220:177-185.

13. Zhang C, Ma L, Niu Y, Wang Z, Xu X, Li Y, Yu Y. Circular rna in lung cancer research: biogenesis, functions, and roles. Int J Biol Sci. 2020; 16:803-814.

14. Wei J, Wei W, Xu H, Wang Z, Gao W, Wang T, Zheng Q, Shu Y, De W. Circular rna hsa_circrna_102958 may serve as a diagnostic marker for gastric cancer. Cancer Biomark. 2020; 27:139-145.

15. Liang G, Ling Y, Mehrpour M, Saw PE, Liu Z, Tan W, Tian Z, Zhong W, Lin W, Luo Q, Lin Q, Li Q, Zhou Y, Hamai A, Codogno P, Li J, Song E, Gong C. Autophagyassociated circrna circcdyl augments autophagy and promotes breast cancer progression. Mol Cancer. 2020; 19:65.

16. Chen X, Mao R, Su W, Yang X, Geng Q, Guo C, Wang Z, Wang J, Kresty LA, Beer DG, Chang AC, Chen G. 
Circular rna circhipk3 modulates autophagy via mir1243p-stat3-prkaa/ampkalpha signaling in stk11 mutant lung cancer. Autophagy. 2020; 16:659-671.

17. Tang XJ, Wang W, Hann SS. Interactions among lncrnas, mirnas and mrna in colorectal cancer. Biochimie. 2019; 163:58-72.

18. Alipoor SD, Adcock IM, Garssen J, Mortaz E, Varahram M, Mirsaeidi M, Velayati A. The roles of mirnas as potential biomarkers in lung diseases. Eur J Pharmacol. 2016; 791:395-404.

19. Wang JY, Chen LJ. The role of mirnas in the invasion and metastasis of cervical cancer. Biosci Rep. 2019; 39:BSR20181377.

20. Hansen TB, Jensen TI, Clausen BH, Bramsen JB, Finsen B, Damgaard CK, Kjems J. Natural rna circles function as efficient microrna sponges. Nature. 2013; 495:384-388.

21. Chen D, Ma W, Ke Z, Xie F. Circrna hsa circ 100395 regulates mir-1228/tcf21 pathway to inhibit lung cancer progression. Cell Cycle. 2018; 17:2080-2090.

22. Cheng Z, Yu C, Cui S, Wang H, Jin H, Wang C, Li B, Qin M, Yang C, He J, Zuo Q, Wang S, Liu J, Ye W, Lv Y, Zhao F, Yao M, Jiang L, Qin W. Circtp63 functions as a cerna to promote lung squamous cell carcinoma progression by upregulating foxm1. Nat Commun. 2019; 10:3200.

23. Clevers H, Nusse R. Wnt/beta-catenin signaling and disease. Cell. 2012; 149:1192-1205.

24. Wang B, Sun L, Li J, Jiang R. miR-577 suppresses cell proliferation and epithelial-mesenchymal transition by regulating the WNT2B mediated Wnt/ $\beta$-catenin pathway in non-small cell lung cancer. Mol Med Rep. 2018; 18:2753-2761

25. Wu Y, Cheng $\mathrm{K}$, Liang W, Wang X. IncRNA RPPH1 promotes non-small cell lung cancer progression through the miR-326/WNT2B axis. Oncol Lett. 2020; 20:105.

Received August 20; Revised October 10, 2020; Accepted October 15, 2020.

\section{*Address correspondence to:}

Baoan Gao, Institute of Respiratory Disease, China Three Gorges University, Yichang Central People's Hospital, Yichang 443002, Hubei, China.

E-mail: jimama39@126.com

Released online in J-STAGE as advance publication November 10, 2020. 